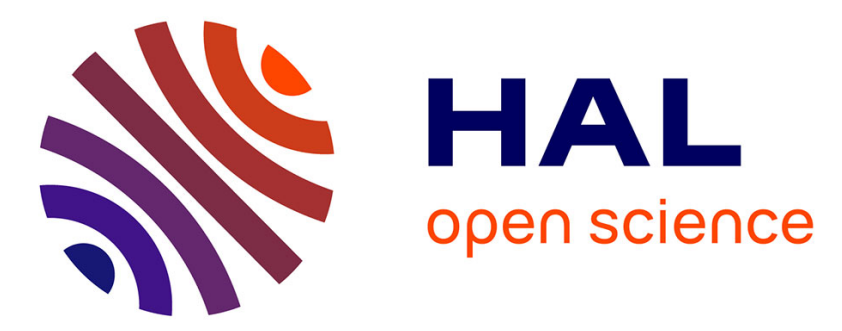

\title{
On Design of Sampled-Data Interval Observers
}

Denis Efimov, Emilia Fridman, Andrey Polyakov, Wilfrid Perruquetti, Jean-Pierre Richard

\section{To cite this version:}

Denis Efimov, Emilia Fridman, Andrey Polyakov, Wilfrid Perruquetti, Jean-Pierre Richard. On Design of Sampled-Data Interval Observers. European Control Conference (ECC16), Mar 2016, Aalborg, Denmark. hal-01295752

\section{HAL Id: hal-01295752 \\ https://hal.inria.fr/hal-01295752}

Submitted on 31 Mar 2016

HAL is a multi-disciplinary open access archive for the deposit and dissemination of scientific research documents, whether they are published or not. The documents may come from teaching and research institutions in France or abroad, or from public or private research centers.
L'archive ouverte pluridisciplinaire HAL, est destinée au dépôt et à la diffusion de documents scientifiques de niveau recherche, publiés ou non, émanant des établissements d'enseignement et de recherche français ou étrangers, des laboratoires publics ou privés. 


\title{
On Design of Sampled-Data Interval Observers
}

\author{
Efimov D., Fridman E., Polyakov A., Perruquetti W., Richard J.-P.
}

\begin{abstract}
New design of interval observers for continuoustime systems with discrete-time measurements is proposed. Positivity and stability conditions are obtained using the timedelay approach. Next, control is synthesized based on interval estimates. Efficiency of the obtained solution is demonstrated on examples.
\end{abstract}

\section{INTRODUCTION}

State estimation in nonlinear systems is a rather complicated and practically important problem [1], [2]. Sparse discrete measurement of the output for a continuous-time plant makes solutions of this problem even more intricate [3], [4], [5], [6], [7]. Especially the observer synthesis is problematical for the cases when the model of a nonlinear system is uncertain, and it contains parametric and/or signal uncertainties. An observer solution for these more complex situations is highly demanded in applications. Interval or set-membership estimation is a promising framework for observation in uncertain systems [8], [9], [10], [11], [12], [13], when all uncertainty is included in the corresponding intervals or polytopes, and as a result the set of admissible values (a Cartesian product of intervals) for the state is provided at each instant of time. The size of that set is related with the level of uncertainty of the plant model.

In this work an interval observer for continuous-discrete systems is proposed. A peculiarity of an interval observer is that in addition to stability conditions, some restrictions on positivity of estimation error dynamics have to be imposed (in order to envelop the system solutions). There exist solutions in the field [14], [15], and in the present work we are going to use the time-delay framework for modeling sampled-data systems [16], [17], [18], [19]. The first objective of this work is to recall the delay-dependent positivity conditions, which are based on the theory of non-oscillatory solutions for functional differential equations [20], [21], and to apply them to continuous-discrete systems. Next, designs of interval observers are given for continuous-time linear systems with discrete measurements (with time-varying sampling). Finally

All authors except E. Fridman are with Non-A team @ INRIA - LNE, Parc Scientifique de la Haute Borne, 40 avenue Halley, 59650 Villeneuve d'Ascq, France and CRIStAL (UMR-CNRS 9189), Ecole Centrale de Lille, BP 48, Cité Scientifique, 59651 Villeneuve-d'Ascq, France.

E. Fridman is with School of Electrical Engineering, Tel-Aviv University, Tel-Aviv 69978, Israel.

D. Efimov is with Department of Control Systems and Informatics, University ITMO, 49 av. Kronverkskiy, 197101 Saint Petersburg, Russia.

This work was partially supported by the Government of Russian Federation (Grant 074-U01), the Ministry of Education and Science of Russian Federation (Project 14.Z50.31.0031) and by Israel Science Foundation (grant No $1128 / 14)$. a control algorithm is proposed based on interval estimates following the ideas of [22].

The paper is organized as follows. Some preliminaries are given in Section 2. The delay-dependent positivity conditions for continuous-discrete systems are presented in Section 3. The interval observer design is performed for a class of linear systems (or a class of nonlinear systems in the output canonical form) with sampled measurements in Section 4. The output control design is carried out in Section 5. Examples of numerical simulation are presented in Section 6.

\section{Notation AND PRELIMINARIES}

In the rest of the paper, the following notation will be used:

- $\mathbb{R}$ is the Euclidean space $\left(\mathbb{R}_{+}=\{\tau \in \mathbb{R}: \tau \geq 0\}\right)$;

- $|x|$ denotes the absolute value of $x \in \mathbb{R},\|x\|$ is the Euclidean norm of a vector $x \in \mathbb{R}^{n}$;

- for a measurable and locally essentially bounded input $u: \mathbb{R}_{+} \rightarrow \mathbb{R}^{p}$ (i.e. for almost all $t \geq 0$ ) the symbol $\|u\|_{\left[t_{0}, t_{1}\right]}$ denotes its $L_{\infty}$ norm $\|u\|_{\left[t_{0}, t_{1}\right]}=$ ess $\sup \left\{\|u(t)\|, t \in\left[t_{0}, t_{1}\right]\right\}$, or simply $\|u\|$ if $t_{0}=0$ and $t_{1}=+\infty$, the set of all such inputs $u \in \mathbb{R}^{p}$ with the property $\|u\|<\infty$ will be denoted as $\mathcal{L}_{\infty}^{p}$;

- for a matrix $A \in \mathbb{R}^{n \times n}$ the vector of its eigenvalues is denoted as $\lambda(A)$;

- $I_{n}$ and $0_{n \times m}$ denote the identity and zero matrices of dimensions $n \times n$ and $n \times m$ respectively;

- $a \mathcal{R} b$ corresponds to an elementwise relation $\mathcal{R}$ ( $a$ and $b$ are vectors or matrices): for example $a<b$ (vectors) means $\forall i: a_{i}<b_{i}$;

- for a symmetric matrix $\Upsilon$, the relation $\Upsilon \prec 0(\Upsilon \preceq 0)$ means that the matrix is negative (semi) definite.

\section{A. Interval bounds}

Given a matrix $A \in \mathbb{R}^{m \times n}$ define $A^{+}=\max \{0, A\}$ (the operation is performed elementwise), $A^{-}=A^{+}-A$ and $|A|=A^{+}+A^{-}$. Let $x \in \mathbb{R}^{n}$ be a vector variable, $\underline{x} \leq x \leq \bar{x}$ for some $\underline{x}, \bar{x} \in \mathbb{R}^{n}$, and $A \in \mathbb{R}^{m \times n}$ be a constant matrix, then [23]:

$$
A^{+} \underline{x}-A^{-} \bar{x} \leq A x \leq A^{+} \bar{x}-A^{-} \underline{x} .
$$

\section{B. General definition of positivity for time-delay systems}

Consider a linear non-autonomous functional differential equation

$$
\begin{gathered}
\dot{x}(t)=\sum_{k=1}^{m} A_{k}(t) x\left[h_{k}(t)\right]+b(t), t \geq t_{0} \geq 0, \\
x(t)=\phi(t) \text { for } t<t_{0}, x\left(t_{0}\right)=x_{0},
\end{gathered}
$$


where $x(t) \in \mathbb{R}^{n}$ is the state vector and $x_{0} \in \mathbb{R}^{n}$; all elements of $A_{k}$ are from $\mathcal{L}_{\infty}$ for all $1 \leq k \leq m, m>0$; the signals $h_{k}:\left[t_{0},+\infty\right) \rightarrow \mathbb{R}$ are Lebesgue measurable and represent the delays $\left(h_{k}(t) \leq t\right.$ and $\lim _{t \rightarrow+\infty} h_{k}(t)=+\infty$ for all $1 \leq k \leq m) ; b \in \mathcal{L}_{\infty}^{n}$ is the input; $\phi:\left(-\infty, t_{0}\right) \rightarrow \mathbb{R}^{n}$ is a Borel measurable bounded function of initial conditions.

Definition 1. For each $s \geq 0$, the solution $\Upsilon(t, s) \in \mathbb{R}^{n \times n}$ of the problem

$$
\begin{gathered}
\frac{\partial \Upsilon(t, s)}{\partial t}=\sum_{k=1}^{m} A_{k}(t) \Upsilon\left[h_{k}(t), s\right], \\
\Upsilon(t, s)=0 \text { for } t<s, \Upsilon(s, s)=I_{n},
\end{gathered}
$$

is called the fundamental (or the Cauchy) matrix of (2).

Lemma 1. [20] (Lemma 9.1) For each $x_{0} \in \mathbb{R}^{n}$ and $\phi$ : $\left(-\infty, t_{0}\right) \rightarrow \mathbb{R}^{n}$ there exists a unique solution of $(2)$, and it can be presented in the form

$$
\begin{aligned}
x(t) & =\Upsilon\left(t, t_{0}\right) x_{0}+\int_{t_{0}}^{t} \Upsilon(t, s) b(s) d s \\
& +\sum_{k=1}^{m} \int_{t_{0}}^{t} \Upsilon(t, s) A_{k}(s) \phi\left[h_{k}(s)\right] d s,
\end{aligned}
$$

where $\phi\left[h_{k}(s)\right]=0$ if $h_{k}(s)>t_{0}$.

Now we are in position to give the definition of positivity, denote $\Omega$ a subset of Borel measurable bounded functions $\phi:\left(-\infty, t_{0}\right) \rightarrow \mathbb{R}^{n}$.

Definition 2. The system (2) for $x_{0} \in \mathbb{R}_{+}^{n}$ and $b(t) \in \mathbb{R}_{+}^{n}$ is called $\Omega$-positive, if $x(t) \geq 0$ for all $t \geq t_{0}$ provided that $\phi \in \Omega$.

A direct consequence of this definition and Lemma 1 is that for $\Omega=\{0\}$ the system is $\{0\}$-positive iff $\Upsilon(t, s) \in$ $\mathbb{R}_{+}^{n \times n}$ for all $t \geq s$.

\section{Delay-dependent positivity}

Consider a scalar time-varying linear system with timevarying delays [20]:

$$
\begin{gathered}
\dot{x}(t)=a_{0}(t) x(t)-a_{1}(t) x[h(t)]+b(t), \\
x(\theta)=0 \text { for } \theta<0, x(0) \in \mathbb{R},
\end{gathered}
$$

where $a_{0} \in \mathcal{L}_{\infty}, a_{1} \in \mathcal{L}_{\infty}, b \in \mathcal{L}_{\infty}, h-t \in \mathcal{L}_{\infty}$ and $h(t) \leq t$ for all $t \geq 0$. For the system (3) the initial condition in (4) is, in general, not a continuous function (if $x(0) \neq 0$ ).

Lemma 2. [20] (Corollary 15.9) Let $0 \leq \frac{1}{e} a_{0}(t) \leq a_{1}(t)$ for all $t \geq 0$ with

$$
\sup _{t \in \mathbb{R}_{+}} \int_{h(t)}^{t}\left[a_{1}(\xi)-\frac{1}{e} a_{0}(\xi)\right] d \xi<\frac{1}{e},
$$

where $e=\exp (1)$. If $x(0) \geq 0$ and $b(t) \geq 0$ for all $t \geq 0$, then the corresponding solution of (3), (4) $x(t) \geq 0$ for all $t \geq 0$.

\section{Positivity OF SAMPLED SYSTEMS}

Consider a time-invariant version of (3) with sampled measurements, i.e.

$$
\begin{gathered}
a_{0}(t)=a_{0}, a_{1}(t)=a_{1}, \\
h(t)=t_{k} \quad \forall t \in\left[t_{k}, t_{k+1}\right), \\
t_{k+1}=t_{k}+T_{k}, k \geq 0, t_{0}=0,
\end{gathered}
$$

where $0<T_{k} \leq \bar{T}$ is a time-varying sampling rate. Then Lemma 2 admits the following corollary.

Corollary 1. For (5), (6) let $0 \leq a_{0} \leq e a_{1}<a_{0}+\bar{T}^{-1}$. If $x(0) \geq 0$ and $b(t) \geq 0$ for all $t \geq 0$, then the corresponding solution of (3), (4) $x(t) \geq 0$ for all $t \geq 0$.

This result can also be extended to the $n$-dimensional system

$$
\dot{x}(t)=A_{0} x(t)-A_{1} x[h(t)]+b(t), t \geq 0,
$$

where $x(t) \in \mathbb{R}^{n}$ is the state; $h(t)$ is defined in (6); $b \in \mathcal{L}_{\infty}^{n}$ is the input; the constant matrices $A_{0}$ and $A_{1}$ have appropriate dimensions. The matrix $A_{0}$ is called Metzler if all its offdiagonal elements are nonnegative.

Proposition 1. Let in (7) $-A_{1}$ be Metzler, $A_{0} \geq 0$, and

$$
\left(A_{0}\right)_{i, i} \leq\left(A_{1}\right)_{i, i} e<\left(A_{0}\right)_{i, i}+\bar{T}^{-1}
$$

for all $i=1, \ldots, n$. If $x(0) \geq 0$ and $b(t) \geq 0$ for all $t \geq 0$, then the corresponding solution of (7) $x(t) \geq 0$ for all $t \geq 0$.

Proof. The result is a consequence of Lemma 2 and Corollary 1.

Let us show how these conditions can be used for design of interval observers.

\section{INTERVAL OBSERVER DESIGN UNDER SAMPLED MEASUREMENTS}

In this section a statement of the problem is given. Next, an interval observer design is presented. And, finally, a control algorithm is synthesized based on interval estimates.

\section{A. Problem statement}

Consider a linear system with sampled measurements:

$$
\begin{aligned}
\dot{x}(t) & =A x(t)+B u(t)+d(t), \\
y(t) & =C x[h(t)]+v[h(t)],
\end{aligned}
$$

where $x(t) \in \mathbb{R}^{n}, h(t)$ is defined in (6); $y(t) \in \mathbb{R}^{p}$ is the system output available for sampled measurements with the noise $v \in \mathcal{L}_{\infty}^{p} ; u \in \mathbb{R}^{m}$ is the control, $u \in \mathcal{L}_{\infty}^{m} ; d \in \mathcal{L}_{\infty}^{n}$ is the system disturbing input; the constant matrices $A, B$ and $C$ have appropriate dimensions. It is assumed that for given $u$ and $d$ the system has a unique solution defined at least locally.

Assumption 1. For given inputs $u \in \mathcal{L}_{\infty}^{m}, d \in \mathcal{L}_{\infty}^{n}$ and initial condition $x(0) \in \mathbb{R}^{n}$, the corresponding solution of 
(8) $x(t)$ is bounded. In addition, there exist known vectors $\underline{x}_{0}, \bar{x}_{0} \in \mathbb{R}^{n}$ such that $\underline{x}_{0} \leq x(0) \leq \bar{x}_{0}$.

Boundedness of the state $x(t)$ is a usual assumption in the estimation theory [1], [2]. The assumption about a known set $\left[\underline{x}_{0}, \bar{x}_{0}\right]$ for the initial conditions $x(0)$ is standard for the interval or set-membership estimation theory [24], [8], [9], [10], [11]. We will assume that the values of matrices $A$, $B$ and $C$ are known, for the sampling $h(t)$ the bound $\bar{T}$ is given:

$$
0 \leq t-h(t) \leq \bar{T},
$$

the instant values of the signals $d(t)$ and $v(t)$ are unavailable. In the last subsection this assumption will be relaxed and a control will be designed ensuring boundedness of the state.

Assumption 2. There exist known signals $\underline{d}, \bar{d} \in \mathcal{L}_{\infty}^{n}$ and $\underline{v}, \bar{v} \in \mathcal{L}_{\infty}^{p}$ such that $\underline{d}(t) \leq d(t) \leq \bar{d}(t)$ and $\underline{v}(t) \leq v(t) \leq$ $\bar{v}(t)$ for all $t \geq 0$.

Therefore, the uncertain inputs $d(t)$ and $v(t)$ in (8) belong to known intervals $[\underline{d}(t), \bar{d}(t)]$ and $[\underline{v}(t), \bar{v}(t)]$, respectively, for all $t \geq 0$.

It is required to design an interval observer,

$$
\begin{gathered}
\dot{\xi}(t)=F[\xi(t), \underline{d}(t), \bar{d}(t), \underline{v}(t), \bar{v}(t), y(t)], \xi(0) \in \mathbb{R}^{s}, \\
\underline{x}(t)=\underline{G}[\xi(t), \underline{d}(t), \bar{d}(t), \underline{v}(t), \bar{v}(t), y(t)], \\
\bar{x}(t)=\bar{G}[\xi(t), \underline{d}(t), \bar{d}(t), \underline{v}(t), \bar{v}(t), y(t)],
\end{gathered}
$$

where $F: \mathbb{R}^{s+2 n+3 p} \rightarrow \mathbb{R}^{s}, \underline{G}: \mathbb{R}^{s+2 n+3 p} \rightarrow \mathbb{R}^{n}$ and $\bar{G}: \mathbb{R}^{s+2 n+3 p} \rightarrow \mathbb{R}^{n}$ are nonlinear maps ensuring existence of solutions, $s>0$, such that $\underline{x}(t) \leq x(t) \leq \bar{x}(t)$ for all $t \geq 0$ and $\underline{x}, \bar{x} \in \mathcal{L}_{\infty}^{n}$. A similar problem has been studied in [14], [15] applying the continuous-discrete framework. In the present work, the time-delay theory is used for this purpose.

\section{B. Motivating example}

Consider a motivating example introduced in [14], where the above problem has been posed for a scalar system

$$
\begin{gathered}
\dot{x}(t)=x(t)+u(t), \\
y(t)=x[h(t)]
\end{gathered}
$$

with $h(t)$ given in (6). This system is unstable for $u(t)=0$. It has been proven in [14] that this system has no interval observer of the form

$$
\begin{aligned}
& \dot{\bar{x}}(t)=-\bar{x}(t)+u(t)+2 y(t), \\
& \underline{\dot{x}}(t)=-\underline{x}(t)+u(t)+2 y(t)
\end{aligned}
$$

(more precisely, the case $u(t)=0$ has been studied in [14]).

Applying the result of Proposition 1, the following interval observer can be proposed for (9) of a form similar to (10).

Claim 1. For the system (9) with any initial condition $x(0) \in$ $\left[\underline{x}_{0}, \bar{x}_{0}\right]$, the system

$$
\begin{gathered}
\underline{\dot{x}}(t)=\underline{x}(t)+u(t)+a\{y(t)-\underline{x}[h(t)]\}, \\
\dot{\bar{x}}(t)=\bar{x}(t)+u(t)+a\{y(t)-\bar{x}[h(t)]\}, \\
\underline{x}(0)=\underline{x}_{0}, \bar{x}(0)=\bar{x}_{0}
\end{gathered}
$$

is an interval observer, i.e. $\underline{x}(t) \leq x(t) \leq \bar{x}(t)$ for all $t \geq 0$, provided that $1 \leq e a<1+\bar{T}^{-1}$.

All proofs are excluded due to space limitations.

For any value of $a$ there exists a sufficiently small $\bar{T}$ such that the conditions of Claim 1 are satisfied. Restricting value of the maximal sampling $\bar{T}$ it is possible to ensure boundedness and asymptotic convergence of errors $\underline{e}$ and $\bar{e}$ [17], [18]. The results of simulation for this observer are given in Section 6.

Let us extend this idea of interval observer design to a more generic system (8).

\section{Interval estimation with sampled measurements}

The equation (8) can be rewritten as follows:

$\dot{x}(t)=A x(t)+B u(t)-L C x[h(t)]+L y(t)+d(t)-L v[h(t)]$,

where $L \in \mathbb{R}^{n \times p}$ is an observer gain to be designed.

Any square matrix $Q$ can be presented as $Q=Q^{\dagger}+Q^{2}$, where $Q^{\dagger}$ is a diagonal matrix composed by all elements on the main diagonals of $Q$, and $Q^{2}$ is formed by the rest elements of $Q$ out of the main diagonal.

Assumption 3. There exists an invertible matrix $S \in \mathbb{R}^{n \times n}$ such that

$$
\begin{gathered}
-S L C S^{-1}=Q_{1}=\left[\begin{array}{cc}
\bar{Q}_{1} & 0_{l \times n-l} \\
0_{n-l \times l} & 0_{n-l \times n-l}
\end{array}\right], \\
S A S^{-1}=Q_{0}=\left[\begin{array}{cc}
Q_{0,1} & Q_{0,2} \\
Q_{0,3} & Q_{0,4}
\end{array}\right], \\
Q_{0,1} \in \mathbb{R}^{l \times l}, Q_{0,2} \in \mathbb{R}^{l \times n-l}, Q_{0,3} \in \mathbb{R}^{n-l \times l}, \\
Q_{0,4} \in \mathbb{R}^{n-l \times n-l}, \bar{Q}_{1}=\bar{Q}_{1}^{\dagger}+\bar{Q}_{1}^{2}, Q_{0,4}=Q_{0,4}^{\dagger}+Q_{0,4}^{2},
\end{gathered}
$$

where $\bar{Q}_{1}^{\dagger}=\operatorname{diag}\left[-q_{1,1}, \ldots,-q_{1, l}\right]$ with $q_{1, k}>0$ for all $k=1, \ldots, l, \bar{Q}_{1}^{2} \geq 0$, and $0<l \leq n$.

This assumption is rather technical and not restrictive, the matrices $S$ and $L$ can be found as a solution of Sylvester equation [13]. In the new coordinates $z=S x=\left[\begin{array}{ll}z_{1}^{\mathrm{T}} & z_{2}^{\mathrm{T}}\end{array}\right]^{\mathrm{T}}$, $z_{1} \in \mathbb{R}^{l}, z_{2} \in \mathbb{R}^{n-l}$ the system (8) takes the form:

$$
\begin{aligned}
\dot{z}_{1}(t)= & \bar{Q}_{0} z(t)+\bar{Q}_{1} z_{1}[h(t)] \\
& +\Sigma_{1} u(t)+\Lambda_{1} y(t)+\delta_{1}(t) \\
\dot{z}_{2}(t)= & Q_{0,3} z_{1}(t)+Q_{0,4} z_{2}(t) \\
& +\Sigma_{2} u(t)+\Lambda_{2} y(t)+\delta_{2}(t)
\end{aligned}
$$

where $\bar{Q}_{0}=\left[\begin{array}{ll}Q_{0,1} & Q_{0,2}\end{array}\right], S B=\left[\begin{array}{ll}\Sigma_{1}^{\mathrm{T}} & \Sigma_{2}^{\mathrm{T}}\end{array}\right]^{\mathrm{T}}$ and $S L=$ $\left[\begin{array}{ll}\Lambda_{1}^{\mathrm{T}} & \Lambda_{2}^{\mathrm{T}}\end{array}\right]^{\mathrm{T}}$ are the matrices of appropriate dimensions; and the input $\delta(t)=\left[\delta_{1}^{\mathrm{T}}(t) \delta_{2}^{\mathrm{T}}(t)\right]^{\mathrm{T}}=S\{d(t)-L v[h(t)]\}$ with the initial condition $z(0)=\left[z_{1}(0)^{\mathrm{T}} z_{2}(0)^{\mathrm{T}}\right]^{\mathrm{T}}=S x(0)$ have interval bounds

$$
\begin{gathered}
\underline{z}_{0} \leq z(0) \leq \bar{z}_{0} \\
\underline{\delta}(t) \leq \delta(t) \leq \bar{\delta}(t) \quad \forall t \geq 0,
\end{gathered}
$$


where

$$
\begin{gathered}
\underline{z}_{0}=S^{+} \underline{x}_{0}-S^{-} \bar{x}_{0}, \bar{z}_{0}=S^{+} \bar{x}_{0}-S^{-} \underline{x}_{0}, \\
\underline{\delta}(t)=S^{+} \underline{d}(t)-S^{-} \bar{d}(t)-(S L)^{+} \bar{v}[h(t)]+(S L)^{-} \underline{v}[h(t)], \\
\bar{\delta}(t)=S^{+} \bar{d}(t)-S^{-} \underline{d}(t)-(S L)^{+} \underline{v}[h(t)]+(S L)^{-} \bar{v}[h(t)] .
\end{gathered}
$$

Then the following interval observer can be proposed for the representation (11):

$$
\begin{aligned}
\dot{\underline{z}}_{1}(t)= & \bar{Q}_{0}^{+} \underline{z}(t)-\bar{Q}_{0}^{-} \bar{z}(t)+\bar{Q}_{1} \underline{z}_{1}[h(t)] \\
& +\Sigma_{1} u(t)+\Lambda_{1} y(t)+\underline{\delta}_{1}(t), \\
\dot{\bar{z}}_{1}(t)= & \bar{Q}_{0}^{+} \bar{z}(t)-\bar{Q}_{0}^{-} \underline{z}(t)+\bar{Q}_{1} \bar{z}_{1}[h(t)] \\
& +\Sigma_{1} u(t)+\Lambda_{1} y(t)+\bar{\delta}_{1}(t), \\
\dot{z}_{2}(t)= & Q_{0,3}^{+} \underline{z}_{1}(t)-Q_{0,3}^{-} \bar{z}_{1}(t)+Q_{0,4}^{\dagger} \underline{z}_{2}(t) \\
& +\left(Q_{0,4}^{2}\right)^{+} \underline{z}_{2}(t)-\left(Q_{0,4}^{2}\right)^{-} \bar{z}_{2}(t) \\
& +\Sigma_{2} u(t)+\Lambda_{2} y(t)+\underline{\delta}_{2}(t), \\
\dot{\bar{z}}_{2}(t)= & Q_{0,3}^{+} \bar{z}_{1}(t)-Q_{0,3}^{-} \underline{z}_{1}(t)+Q_{0,4}^{\dagger} \bar{z}_{2}(t) \\
& +\left(Q_{0,4}^{2}\right)^{+} \bar{z}_{2}(t)-\left(Q_{0,4}^{2}\right)^{-} \underline{z}_{2}(t) \\
& +\Sigma_{2} u(t)+\Lambda_{2} y(t)+\bar{\delta}_{2}(t),
\end{aligned}
$$

with initial conditions $\underline{z}(0)=\underline{z}_{0}, \bar{z}(0)=\bar{z}_{0}$ for the variables $\underline{z}(t)=\left[\begin{array}{ll}\underline{z}_{1}^{\mathrm{T}}(t) & \underline{z}_{2}^{\mathrm{T}}(t)\end{array}\right]^{\mathrm{T}}, \bar{z}(t)=\left[\begin{array}{ll}\bar{z}_{1}^{\mathrm{T}}(t) & \bar{z}_{2}^{\mathrm{T}}(t)\end{array}\right]^{\mathrm{T}}$ respectively. Finally interval estimates for the variable $x(t)$ can be obtained using

$$
\begin{aligned}
& \underline{x}(t)=\left(S^{-1}\right)^{+} \underline{z}(t)-\left(S^{-1}\right)^{-} \bar{z}(t), \\
& \bar{x}(t)=\left(S^{-1}\right)^{+} \bar{z}(t)-\left(S^{-1}\right)^{-} \underline{z}(t) .
\end{aligned}
$$

For all $k=1, \ldots, l$ denote

$$
q_{0, k}=\left(\bar{Q}_{0}^{+}\right)_{k, k} \text {. }
$$

Theorem 1. Let assumptions 1, 2 and 3 be satisfied and

$$
q_{0, k} \leq e q_{1, k}<q_{0, k}+\bar{T}^{-1}
$$

for all $k=1, \ldots, l$. Then the interval observer (12), (13) for the system (8), (6) admits the relations

$$
\underline{x}(t) \leq x(t) \leq \bar{x}(t) \quad \forall t \geq 0 .
$$

If in addition there exist symmetric matrices $P \in \mathbb{R}^{2 n \times 2 n}$ and $W \in \mathbb{R}^{2 n \times 2 n}$ such that the LMIs

$$
\begin{aligned}
& {\left[\begin{array}{ccc}
\Psi & \frac{2 \bar{T}}{\pi} P \Phi_{1} & \left(\Phi_{0}+\Phi_{1}\right)^{T} W \\
\frac{2 \bar{T}}{\pi} \Phi_{1}^{T} P & -W & \frac{2 \bar{T}}{\pi} \Phi_{1}^{T} W \\
W\left(\Phi_{0}+\Phi_{1}\right) & \frac{2 \bar{T}}{\pi} W \Phi_{1} & -W
\end{array}\right] \prec 0} \\
& P \succ 0, W \succ 0, \\
& \Psi=P\left(\Phi_{0}+\Phi_{1}\right)+\left(\Phi_{0}+\Phi_{1}\right)^{T} P
\end{aligned}
$$

are satisfied for

$$
\begin{gathered}
\Phi_{0}=\left[\begin{array}{cccc}
Q_{01}^{+} & -Q_{01}^{-} & Q_{02}^{+} & -Q_{02}^{-} \\
-Q_{01}^{-} & Q_{01}^{+} & -Q_{02}^{-} & Q_{02}^{+} \\
Q_{0,3}^{+} & -Q_{0,3}^{-} & Q_{0,4}^{\dagger}+\left(Q_{0,4}^{2}\right)^{+} & -\left(Q_{0,4}^{2}\right)^{-} \\
-Q_{0,3}^{-} & Q_{0,3}^{+} & -\left(Q_{0,4}^{2}\right)^{-} & Q_{0,4}^{\dagger}+\left(Q_{0,4}^{2}\right)^{+}
\end{array}\right], \\
\Phi_{1}=\left[\begin{array}{cccc}
\bar{Q}_{1} & 0_{l \times l} & 0_{l \times n-l} & 0_{l \times n-l} \\
0_{l \times l} & \bar{Q}_{1} & 0_{l \times n-l} & 0_{l \times n-l} \\
0_{n-l \times l} & 0_{n-l \times l} & 0_{n-l \times n-l} & 0_{n-l \times n-l} \\
0_{n-l \times l} & 0_{n-l \times l} & 0_{n-l \times n-l} & 0_{n-l \times n-l}
\end{array}\right],
\end{gathered}
$$

then $\underline{x}, \bar{x} \in \mathcal{L}_{\infty}^{n}$.

In order to prove boundedness of $\underline{x}, \bar{x}$ a Lyapunov functional candidate

$$
\begin{aligned}
& V(t)=\zeta^{\mathrm{T}}(t) P \zeta(t)+\bar{T}^{2} \int_{t_{k}}^{t} \dot{\zeta}^{\mathrm{T}}(s) W \dot{\zeta}(s) d s \\
& -\frac{\pi^{2}}{4} \int_{t_{k}}^{t}\left[\zeta(s)-\zeta\left(t_{k}\right)\right]^{\mathrm{T}} W\left[\zeta(s)-\zeta\left(t_{k}\right)\right] d s
\end{aligned}
$$

for $t_{k} \leq t<t_{k+1}$ from [18] has been considered, where $\zeta=\left[\begin{array}{llll}\underline{z}_{1}^{T} & \bar{z}_{1}^{T} & \underline{z}_{2}^{T} & \bar{z}_{2}^{T}\end{array}\right]^{T}$.

\section{OUTPUT STABILIZING CONTROL BASED ON INTERVAL ESTIMATES}

Up to now we supposed that the state $x(t)$ and the control $u(t)$ are already given and they are bounded. However, the interval estimates $\underline{x}(t), \bar{x}(t)$ can be effectively used to stabilize the uncertain system (8) [22]. Indeed, the interval observer (12), (13) guarantees interval inclusion (14) for any input $u(t)$. If a control $u(t)$ is designed such that both variables $\underline{x}(t), \bar{x}(t)$ are bounded and converge to zero, due to (14), $x(t)$ will possess the same properties. Therefore, it is possible to substitute the problem of output stabilizing control design of uncertain system (8) by the problem of state stabilizing feedback design for completely known observer (12). In this case the observer gain $L$ has to ensure positivity of the estimation errors $\bar{e}, \underline{e}$ only, and boundedness of all variables can be provided by a proper control design. Therefore, the gain $L$ has to ensure validity of Assumption 3 (solution of a Sylvester equation), while stability LMIs (15) are provided by control selection.

Let us restrict our attention to the case of sampled control, i.e. $u(t)=u[h(t)]$ where $h(t)$ is defined in (6). According to (13), stabilization of $\underline{x}(t), \bar{x}(t)$ follows stabilization of $\underline{z}(t)$, $\bar{z}(t)$, then the following feedback is appropriate due to the structure of (12):

$$
u=\underline{K z}+\bar{K} \bar{z}
$$

where $\underline{K} \in \mathbb{R}^{m \times n}, \bar{K} \in \mathbb{R}^{m \times n}$ are the control gains to be designed. For $\underline{K}=\left[\underline{K}_{1} \underline{K}_{2}\right]$ and $\bar{K}=\left[\bar{K}_{1} \bar{K}_{2}\right]$, where $\underline{K}_{1}$, $\underline{K}_{2}, \bar{K}_{1}$ and $\bar{K}_{2}$ have appropriate dimensions, (12) can be rewritten as follows:

$$
\dot{\zeta}(t)=\Phi_{0} \zeta(t)+\Phi_{2} \zeta[h(t)]+\Lambda y(t)+\delta(t),
$$

where $\zeta=\left[\begin{array}{llll}z_{1}^{T} & \bar{z}_{1}^{T} & \underline{z}_{2}^{T} & \bar{z}_{2}^{T}\end{array}\right]^{T}$ and $\Phi_{0}$ are as before,

$$
\Phi_{2}=\Phi_{1}+\Sigma\left[\underline{K}_{1} \bar{K}_{1} \underline{K}_{2} \bar{K}_{2}\right] .
$$

Note that for stabilization of $\underline{x}(t), \bar{x}(t)$ (or $\zeta(t)$ ) the signal $y(t)$ is a kind of functional perturbation, which is globally Lipschitz with respect to $\zeta(t)$. Indeed, from the relation 
$\underline{x}(t) \leq x(t) \leq \bar{x}(t)$ we have

$$
\begin{aligned}
\left|x_{i}(t)\right| & \leq \max \left\{\left|\underline{x}_{i}(t)\right|,\left|\bar{x}_{i}(t)\right|\right\} \quad \forall 1 \leq i \leq n, \\
\left|x_{i}(t)\right|^{2} & \leq\left|\underline{x}_{i}(t)\right|^{2}+\left|\bar{x}_{i}(t)\right|^{2} \quad \forall 1 \leq i \leq n \\
\|x(t)\| & \leq \sqrt{\|\underline{x}(t)\|^{2}+\|\bar{x}(t)\|^{2}} \\
& \leq \mu \sqrt{\|\underline{z}(t)\|^{2}+\|\bar{z}(t)\|^{2}} \\
& \leq \mu\|\zeta\|,
\end{aligned}
$$

where $\mu=\sqrt{2\left[\left\|\left(S^{-1}\right)^{+}\right\|^{2}+\left\|\left(S^{-1}\right)^{-}\right\|^{2}\right]}$, then $\|y(t)\| \leq$ $\|C\| \mu\|\zeta[h(t)]\|$.

Theorem 2. Let assumptions 2 and 3 be satisfied, $\underline{x}_{0} \leq$ $x(0) \leq \bar{x}_{0}$ for some known vectors $\underline{x}_{0}, \bar{x}_{0} \in \mathbb{R}^{n}$, and

$$
q_{0, k} \leq e q_{1, k}<q_{0, k}+\bar{T}^{-1}
$$

for all $k=1, \ldots, l$. Then the interval observer (12), (13) for the system (8), (6) admits the relations (14). If in addition there exist symmetric matrices $P \in \mathbb{R}^{2 n \times 2 n}$ and $U \in$ $\mathbb{R}^{2 n \times 2 n}$, and matrices $X, X_{1}, P_{2}, P_{3}, R, Y_{1}, Y_{2} \in \mathbb{R}^{2 n \times 2 n}$ such that the LMIs

$$
\begin{aligned}
& {\left[\begin{array}{cc}
\bar{T}^{-1} P+\frac{X+X^{T}}{2} & X_{1}-X \\
* & \frac{X+X^{T}}{2}-\left[X_{1}+X_{1}^{T}\right]
\end{array}\right] \succ 0,} \\
& {\left[\begin{array}{c|ccc} 
& \mid & P_{2}^{T} \Lambda & 0_{2 n \times 2 n} \\
\Psi_{i} & & P_{3}^{T} \Lambda & 0_{2 n \times 2 n} \\
& & 0_{2 n \times 2 n} & 0_{2 n \times 2 n} \\
& & 0_{2 n \times 2 n} & I_{2 n} \\
* & - & -\gamma^{2} I_{2 n} & - \\
* & & - & 0_{2 n \times 2 n} \\
* & & * & -I_{2 n}
\end{array}\right] \prec 0, i=0,1,} \\
& P \succ 0, U \succ 0, \\
& \Psi_{0}=\left[\begin{array}{ccc}
\Omega_{11}-\frac{X+X^{T}}{2} & \Omega_{12}+\bar{T} \frac{X+X^{T}}{2} & \Omega_{13}+X-X_{1} \\
* & \Omega_{22}+\bar{T} U & \Omega_{23}-\bar{T}\left(X-X_{1}\right) \\
* & * & \Omega_{33}-\Pi
\end{array}\right], \\
& \Psi_{1}=\left[\begin{array}{cccc}
\Omega_{11}-\frac{X+X^{T}}{2} & \Omega_{12} & \Omega_{13}+X-X_{1} & \bar{T} Y_{1}^{T} \\
* & \Omega_{22} & \Omega_{23} & \bar{T} Y_{2}^{T} \\
* & * & \Omega_{33}-\Pi & \bar{T} R^{T} \\
* & * & * & -\bar{T} U
\end{array}\right] \\
& \Omega_{11}=\Phi_{0}^{T} P_{2}+P_{2}^{T} \Phi_{0}-Y_{1}-Y_{1}^{T}, \\
& \Omega_{12}=P-P_{2}^{T}+\Phi_{0}^{T} P_{3}-Y_{2}, \\
& \Omega_{13}=Y_{1}^{T}+P_{2}^{T} \Phi_{2}-R, \\
& \Omega_{22}=-P_{3}-P_{3}^{T}, \Omega_{23}=Y_{2}^{T}+P_{3}^{T} \Phi_{2}, \\
& \Omega_{33}=R+R^{T}, \Pi=\frac{X+X^{T}-2\left(X_{1}+X_{1}^{T}\right)}{2}
\end{aligned}
$$

are satisfied for $\gamma<\frac{1}{|C| \mid \mu}$, then $x, \underline{x}, \bar{x} \in \mathcal{L}_{\infty}^{n}$.

The main advantage of Theorem 2 with respect to Theorem 1 is that its conditions are decoupled: the observer gain $L$ ensures positivity of the estimation error dynamics, while the control gains $\underline{K}, \bar{K}$ guarantee stability.

Let us demonstrate efficiency of the developed approach for interval control and output stabilization on examples.

\section{EXAMPLES}

A. Observer and control for the motivating example

The system (9) is an example of (8) for $n=1$

$$
\dot{x}(t)=x(t)+u(t)+d(t), y(t)=x[h(t)]+v[h(t)],
$$
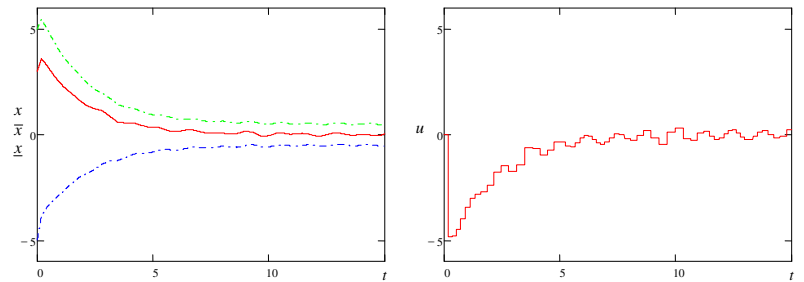

Figure 1. The results of simulation for the motivating example

where $d(t) \in[-0.1,0.1]$ (for simulation $d(t)=0.1 \cos (3 t)$ ), $v(t) \in[-0.1,0.1]$ (for simulation $v(t)=0.1 \sin (5 t)$ ) and $T_{k}=\frac{\bar{T}}{2}\left(1+\sin ^{2}\left(0.5 t_{k}\right)\right)$ with $\bar{T}=0.35$. Note that $e^{-1}(1+$ $\left.\bar{T}^{-1}\right)=1.419$, then select:

$$
L=1.4, \underline{K}=-3, \bar{K}=-3
$$

and assume that $\left\|x_{0}\right\| \leq 5$. The interval observer (12) takes a form similar to one given in Claim 1. The results of simulation are shown in Fig. 1. The red solid curve represents a trajectory of the system, the blue and green dash-dot lines correspond to the interval estimates $\underline{x}(t)$ and $\bar{x}(t)$ generated by the observer.

\section{B. A pendulum example}

Consider an example of (8) for $n=2$ from [15]

$$
\begin{gathered}
A=\left[\begin{array}{cc}
0 & 1 \\
-1 & 0
\end{array}\right], B=\left[\begin{array}{l}
0 \\
1
\end{array}\right], d(t)=\delta\left[\begin{array}{c}
\sin (t) \\
\cos (2 t)
\end{array}\right], \\
C=\left[\begin{array}{ll}
1 & 0
\end{array}\right], v(t)=V \sin (5 t), \bar{T}=0.1,
\end{gathered}
$$

where $\delta>0$ and $V>0$ are the upper bounds of $d$ and $v$ available for designer. Thus, Assumption 2 is satisfied for:

$$
\begin{gathered}
\underline{d}(t)=-\left[\begin{array}{l}
\delta \\
\delta
\end{array}\right], \bar{d}(t)=-\underline{d}(t), \\
\bar{v}(t)=-\underline{v}(t)=V .
\end{gathered}
$$

Take $\left\|x_{10}\right\| \leq 10,\left\|x_{20}\right\| \leq 10$. For $L=[44]^{T}$ and

$$
S=\left[\begin{array}{cc}
\sqrt{2} & 0 \\
-1 & 1
\end{array}\right]
$$

the conditions of Assumption 3 are verified. The LMIs of Theorem 1 are satisfied for the given value of $\bar{T}$, the conditions of Theorem 2 are also satisfied for

$$
\underline{K}=-\frac{1}{8}\left[\begin{array}{ll}
1 & 1
\end{array}\right], \bar{K}=-\frac{1}{16}\left[\begin{array}{ll}
1 & 1
\end{array}\right] .
$$

The results of simulation are shown in Fig. 2 for

$$
\delta=0.5, V=0.1, T_{k}=\bar{T}\left[0.5+0.4 \sin \left(0.5 t_{k}\right)\right],
$$

they confirm efficiency of interval estimation and validity of used delay-dependent positivity conditions. 

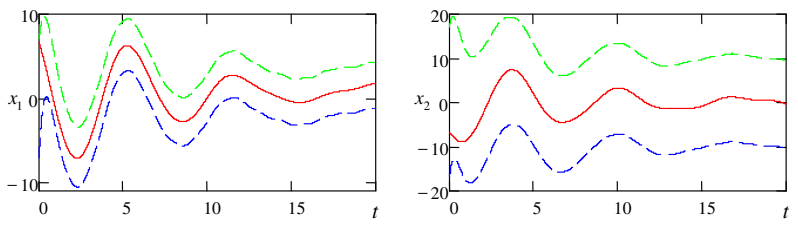

Figure 2. The results of simulation for the sampled pendulum

\section{CONCLUSION}

In the paper, new positivity conditions for linear sampled systems have been proposed. These conditions are related with non-oscillatory behavior of solutions of the corresponding time-delay representation [20]. These new conditions have been employed to design interval observer for the systems with sampled measurements extending the theory of [14], [15]. The results have been applied for the benchmark system from [14]. An output stabilizing control has been proposed based on interval state estimates. The efficacy of observers has been illustrated by numerical experiments. The problem of optimal selection of the observer gains and handling parametric uncertainty of the model are directions of future work.

\section{REFERENCES}

[1] G. Besançon, Ed., Nonlinear Observers and Applications, ser. Lecture Notes in Control and Information Sciences. Springer, 2007, vol. 363.

[2] T. Fossen and H. Nijmeijer, New Directions in Nonlinear Observer Design. Springer, 1999.

[3] V. Andrieu and M. Nadri, "Observer design for Lipschitz systems with discrete-time measurements," in Proc. 49th IEEE Conference on Decision and Control, Atlanta, 2010, pp. 6522-6527.

[4] D. Deza, E. Busvelle, J. P. Gauthier, and D. Rakotopara, "High gain estimation for nonlinear systems," Systems \& Control Letters, vol. 18, no. 4, pp. 295-299, 1992.

[5] G. Goffaux, A. Vande Wouwer, and O. Bernard, "Improving continuous-discrete interval observers with application to microalgaebased bioprocess," Journal of Process Control, vol. 19, no. 7, pp. 1182 $1190,2009$.

[6] H. Hammouri, N. Nadri, and R. Mota, "Constant gain observer design for continuous-discrete time uniformly observable systems," in Proc. 45th IEEE Conference on Decision and Control, San Diego, CA, 2006, pp. 5406-5411.

[7] M. Nadri, H. Hammouri, and C. Astorga, "Observer design for continuous discrete-time state affine systems up to output injection," European Journal of Control, vol. 10, no. 3, pp. 252-262, 2004.

[8] J. Gouzé, A. Rapaport, and M. Hadj-Sadok, "Interval observers for uncertain biological systems," Ecological Modelling, vol. 133, pp. 4656, 2000.

[9] L. Jaulin, "Nonlinear bounded-error state estimation of continuous time systems," Automatica, vol. 38, no. 2, pp. 1079-1082, 2002.

[10] M. Kieffer and E. Walter, "Guaranteed nonlinear state estimator for cooperative systems," Numerical Algorithms, vol. 37, pp. 187-198, 2004.

[11] M. Moisan, O. Bernard, and J. Gouzé, "Near optimal interval observers bundle for uncertain bio-reactors," Automatica, vol. 45, no. 1, pp. 291295, 2009.

[12] F. Mazenc and O. Bernard, "Interval observers for linear time-invariant systems with disturbances," Automatica, vol. 47, no. 1, pp. 140-147, 2011.

[13] T. Raïssi, D. Efimov, and A. Zolghadri, "Interval state estimation for a class of nonlinear systems," IEEE Trans. Automatic Control, vol. 57, no. 1, pp. 260-265, 2012.
[14] F. Mazenc, M. Kieffer, and E. Walter, "Interval observers for continuous-time linear systems with discrete-time outputs," in Proc 2012 American Control Conference, Montreal, 2012, pp. 1889-1894.

[15] F. Mazenc and T. Dinh, "Construction of interval observers for continuous-time systems with discrete measurements," Automatica, vol. 50, no. 10, pp. 2555-2560, 2014.

[16] E. Fridman, A. Seuret, and J.-P. Richard, "Robust sampled-data stabilization of linear systems: an input delay approach," Automatica, vol. 40, pp. 1441-1446, 2004.

[17] E. Fridman, "A refined input delay approach to sampled-data control," Automatica, vol. 46, pp. 421-427, 2010.

[18] K. Liu and E. Fridman, "Wirtinger's inequality and Lyapunov-based sampled-data stabilization," Automatica, vol. 48, pp. 102-108, 2012.

[19] E. Fridman, Introduction to Time-Delay Systems: Analysis and Control. Basel: Birkhäuser, 2014.

[20] R. P. Agarwal, L. Berezansky, E. Braverman, and A. Domoshnitsky, Nonoscillation theory of functional differential equations with applications. New York: Springer, 2012.

[21] A. Domoshnitsky, "Maximum principles and nonoscillation intervals for first order Volterra functional differential equations," Dynamics of Continuous, Discrete \& Impulsive Systems. A: Mathematical Analysis, vol. 15, pp. 769-814, 2008.

[22] D. Efimov, T. Raïssi, and A. Zolghadri, "Control of nonlinear and LPV systems: interval observer-based framework," IEEE Trans. Automatic Control, vol. 58, no. 3, pp. 773-782, 2013.

[23] D. Efimov, L. Fridman, T. Raïssi, A. Zolghadri, and R. Seydou, "Interval estimation for LPV systems applying high order sliding mode techniques," Automatica, vol. 48, pp. 2365-2371, 2012.

[24] D. Efimov, W. Perruquetti, and J.-P. Richard, "Interval estimation for uncertain systems with time-varying delays," International Journal of Control, vol. 86, no. 10, pp. 1777-1787, 2013. 